\title{
The Quest for Solving QCD: Simulations at Light Quark Masses ${ }^{1}$
}

\author{
K. Jansen \\ NIC, DESY, Platanenallee 6, 15738 Zeuthen, Germany
}

\begin{abstract}
We present lattice QCD simulation results from the European Twisted Mass Collaboration (ETMC). In particular, we show the strange baryon spectrum, list a number of precisely determined low energy constants of chiral perturbation theory and provide a first account of simulations including the strange and charm degrees of freedom.
\end{abstract}

DOI: $10.1134 / \mathrm{S} 1063779610060079$

\section{INTRODUCTION}

We strongly believe nowadays that the quarks are the constituents of all hadrons with the gluons being the interaction particles that -via the strong interaction- "glue" the quarks together to form the bound hadron states which are the ones observed in experiments. The mechanism of forming the bound states is theoretically described by quantum chromodynamics. The postulation of QCD is that at very short distances the quarks behave as almost free particles that interact only very weakly, a phenomenon we call asymptotic freedom. At large distances, at the order of $1 \mathrm{fm}$, the quarks interact extremely strong and in fact so strong that they will never be seen as final, observable states but rather form the observed hadron bound spectrum. The latter phenomenon is called confinement of quarks.

Since the interaction between quarks become so strong at large distances, analytical methods such as perturbation theory fail to analyze QCD. A method to nevertheless tackle the problem is to formulate QCD on a 4-dimensional, euclidean space-time grid. This setup first of all allows for a rigorous definition of QCD and leads to fundamental theoretical and conceptual investigations. On the other hand, the lattice approach enables theorists to perform large scale numerical simulations. In this contribution, we will describe one approach to "lattice QCD", the twisted mass formulation.

In the past, lattice physicists had to work with a number of limitations when performing numerical simulations. These simulations are extremely expensive, reaching the need for Petaflop computing and even beyond, a regime of computing power we just reach today. Therefore, for a long time the quarks were treated as infinitely heavy, indeed a crude approximation given that the up and down quarks have masses of only $\mathrm{O}(\mathrm{MeV})$. In a next step, only the lightest quark doublet, the up and down quarks, were taken into con-

\footnotetext{
${ }^{1}$ The article is published in the original.
}

sideration, although their mass values as used in the simulation had been unphysically large.

Nowadays, besides the up and down quarks, also the strange quark is included in the simulations. In addition, these simulations are performed in almost physical conditions, having the quark masses close to their physical values, large lattices with about $3 \mathrm{fm}$ linear extent and small values of the lattice spacing such that a controlled continuum limit can be performed. The situation of the change of the simulation landscape is illustrated in fig. 1a. In the figure, the blue dot indicates the physical point. The black cross represents a state of the art simulation in the year 2001. As can be seen in the graph, most of the simulations now go well beyond what could be reached in 2001 demonstrating clearly the progress in performing realistic simulations.

The drastic change in the situation is due to three main developments: (i) algorithmic breakthroughs; (ii) machine development; the computing power of the present $\mathrm{BG} / \mathrm{P}$ systems is even outperforming Moore's law, (iii) conceptual developments, such as the use of improved actions which reduce lattice artefacts and the development of non-perturbative renormalization.

As a physical example of results we can achieve presently, we show in Fig. 1b the continuum extrapolated strange baryon spectrum as obtained [1] by the European Twisted Mass Collaboration (ETMC) of which the author is a member. ETMC comprises 16 institutions in Europe, i.e. Cyprus (Univ. of Cyprus), France (Univ. of Paris Sud and LPSC Grenoble), Germany (Humboldt Univ. zu Berlin, Univ. of Münster, DESY in Hamburg and Zeuthen), Great Britain (Univ. of Glasgow and Univ. of Liverpool), Italy (Univ. of Rome I, II and III, ECT* Trento), Netherlands (Univ. of Groningen), Poland, (Univ. of Poznan), Spain (Univ. of Valencia), Switzerland (Univ. of Bern).

The baryon spectrum calculation has been considered a benchmark study for lattice QCD for a long 

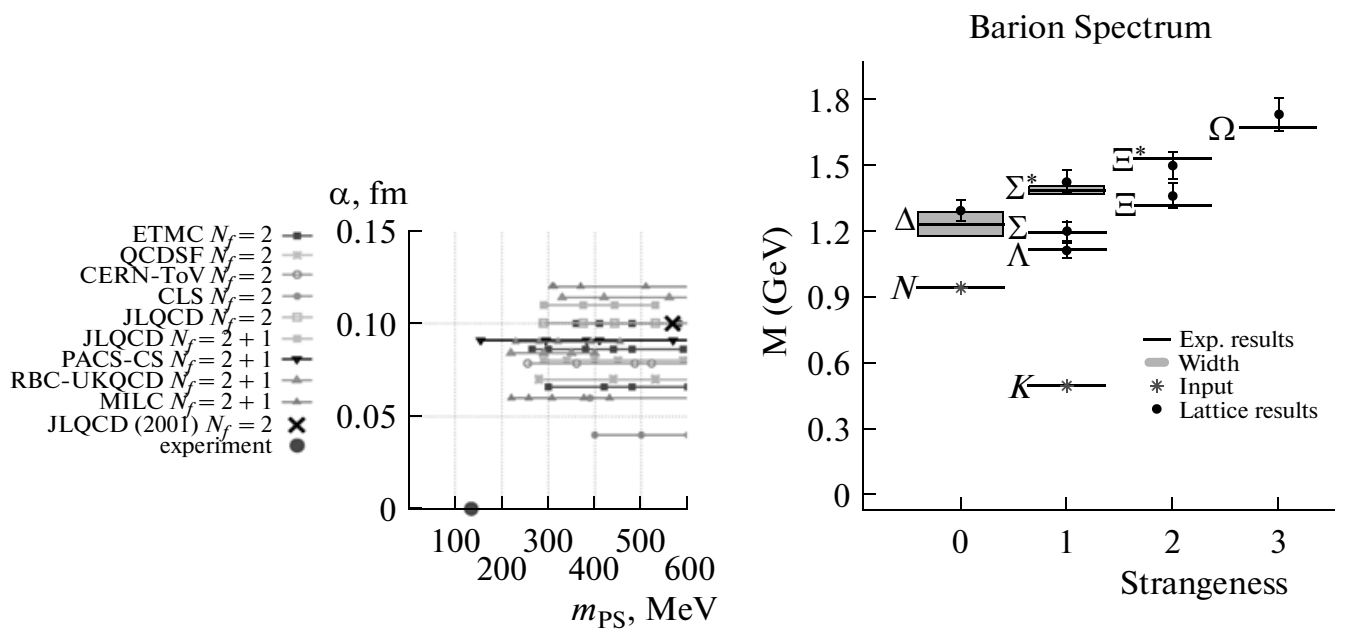

Fig. 1. (a) The values of the lattice spacing a and pseudo scalar masses $m_{\mathrm{PS}}$ as employed presently in typical QCD simulations by various collaborations as (incompletely) listed in the legend. The blue dot indicates the physical point where in the continuum the pseudo scalar meson assumes its experimentally measured value. The black cross represents a state of the art simulation by the JLQCD collaboration in 2001. (b) The continuum strange baryon spectrum from the ETM collaboration.

time. It is therefore very reassuring that finally this important result can be obtained precisely from abinitio and non-perturbative lattice simulations.

\section{TWISTED MASS FERMIONS}

Twisted mass fermions [3, 4] belong to the class of Wilson fermions [2]. In this approach the lattice artefacts in physical observables appear only quadratic in the lattice spacing. This is in contrast to a standard Wilson action, where these lattice spacing effects are linear. The main advantage of the twisted mass formulation of lattice QCD is then that these kind of fermions provide an improved, i.e. $\mathrm{O}\left(a^{2}\right)$, continuum limit scaling of physical observables. The twisted mass formulation of Lattice QCD [3, 4] is being studied extensively with $N_{f}=2$ dynamical flavours, i.e. including only the lightest up and down quark doublet, by the European Twisted Mass collaboration, see [8] and references therein.

The fermionic action for two flavours of twisted, mass degenerate quarks in the so called twisted basis $[3,9]$ reads

$$
S_{\mathrm{tm}}=a^{4} \sum_{x}\left\{\bar{\chi}(x)\left[D[U]+m_{0}+i \mu_{q} \gamma_{5} \tau^{3}\right] \chi(x)\right\},
$$

where $m_{0}$ is the untwisted bare quark mass, $\mu_{q}$ is the bare twisted quark mass, $\tau^{3}$ is the third Pauli matrix acting in flavour space and

$$
D[U]=\frac{1}{2}\left[\gamma_{\mu}\left(\nabla_{\mu}+\nabla_{\mu}^{*}\right)-a \nabla_{\mu}^{*} \nabla_{\mu}\right]
$$

is the mass-less Wilson-Dirac operator. $\nabla_{\mu}$ and $\nabla_{\mu}^{*}$ are the forward and backward gauge covariant differ- ence operators, respectively. Twisted mass fermions are said to be at maximal twist if the bare untwisted quark mass $m_{0}$ is tuned to its critical value $m_{\text {crit }}$, the situation we shall be interested in. For convenience we define the hopping parameter $\kappa=1 /\left(8+2 a m_{0}\right)$.

Maximally twisted mass fermions provide important advantages: the spectrum of $Q^{\dagger} Q$ with $Q=\gamma_{5}(D[U]+$ $\left.m_{0}+i \mu_{q} \gamma_{5}\right)$ is bounded from below, which was the original reason to consider twisted mass fermions [3]. At maximal twist, the twisted quark mass $\mu_{q}$ is related directly to the physical quark mass and renormalises multiplicatively only. Many mixings under renormalisation are expected to be simplified [4, 9]. And, physical observables are automatically $O(a)$ improved. Another feature of maximally twisted mass fermions is that the pseudo scalar decay constant $f_{\mathrm{PS}}$ does not need any renormalisation which allows for a very precise determination of this quantity.

The main drawback of maximally twisted mass fermions is that both parity and flavour symmetry are broken explicitly at non-zero values of the lattice spacing. However, it turns out that this is presumably only relevant for the mass of the neutral pseudo scalar meson (and kinematically related quantities) [10].

\section{RESULTS FOR TWO FLAVOURS OF MASS-DEGENERATE QUARKS}

Since in the maximal twist situation the theory is O (a)-improved, leading lattice artefacts are expected to be of order $a^{2}$. This can be checked by extrapolating a physical quantity in units of the force parameter [11] $r_{0}^{\chi}$ extrapolated to the chiral limit at fixed physical situa- 

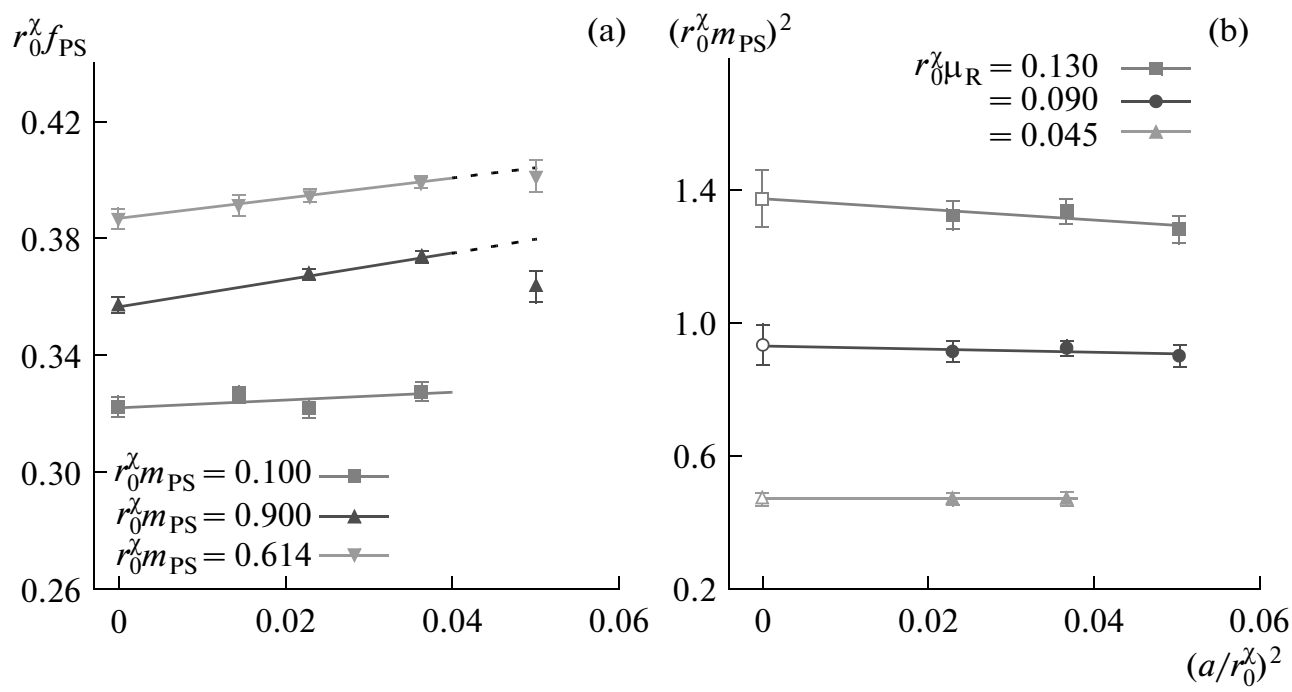

Fig. 2. Scaling in finite, fixed volume for $r_{0}^{\chi} f_{\mathrm{PS}}$ at fixed values of $r_{0}^{\chi} m_{\mathrm{PS}}$ (a) and for $\left(r_{0}^{\chi} m_{\mathrm{PS}}\right)^{2}$ at fixed values of $r_{0}^{\chi} \mu_{R}$ (b). In (b) we cannot include data at $\beta=4.2$ due to the missing value of the renormalisation factor $Z_{\mathrm{P}}$.

tion to the continuum limit. We show two such examples in Fig. 2. In the left panel we show $r_{0}^{\chi} f_{\mathrm{PS}}$ as a function of $\left(a / r_{0}^{\chi}\right)^{2}$ at fixed value of $r_{0}^{\chi} m_{\mathrm{PS}}$. In order to match the values of $r_{0}^{\chi} m_{\mathrm{PS}}$ at each value of $r_{0}^{\chi} / a$ and to fix the volume to $r_{0}^{\chi} \cdot L=5$ we had to perform short inter- or extra-polations. The straight lines are linear fits in $\left(a / r_{0}^{\chi}\right)^{2}$ to the corresponding data, with the data at the largest value of the lattice spacing not being included in the fit. It is clearly visible that the lattice artefacts appear to scale linearly in $a^{2}$ and that their overall size is small.

In the right panel of Fig. 2 we show the scaling of $r_{0}^{\chi} m_{\mathrm{PS}}$ as a function of $\left(a / r_{0}^{\chi}\right)^{2}$ at fixed values of the renormalised quark mass $r_{0}^{\chi} \mu_{R}$, again at fixed, finite volume. We conclude that also the charged pseudo scalar mass has only small lattice artefacts.

The dependence of $m_{\mathrm{PS}}$ and $f_{\mathrm{PS}}$ on the renormalised quark mass and volume can be described by chiral perturbation theory $(\chi \mathrm{PT})[13,14]$. The residual lattice artefacts of order $a^{2}$ can also be included in the analysis. The corresponding formulae can be found in $[8,15]$. We fit these formulae to our data in order to extract the parameters of the $N_{f}=2$ chiral Lagrangian, i.e. the low energy constants and some derived quantities. Moreover, we can use these fits to calibrate our lattices by determining the value of the renormalised quark mass $r_{0}^{\chi} \mu_{R}$ where the ratio $m_{\mathrm{PS}} / f_{\mathrm{PS}}$ assumes its physical value (i.e. $m_{\pi} / f_{\pi}$ ) and set $f_{\mathrm{PS}}=f_{\pi}=130.7 \mathrm{MeV}$ there, as done in [5]. Hence, $f_{\pi}$ is used in this paper to set the scale.
The results of these fits can be found in Table 3. We give statistical and systematic errors separately, the systematic one being asymmetrical. The results are obtained by performing $\mathcal{O}(80)$ fits, which differ in fitrange, finite size correction formulae and in the order of $\chi$ PT. The final result is obtained as the median of the corresponding weighted distribution over all fits. The statistical error is determined using the bootstrap method with 1000 samples. The systematic uncertainty is estimated from the $68 \%$ confidence interval of the weighted distribution. For details see [8]. The fit results for the determination of physical quantities and

Summary of fit results, determined from the weighted distribution of a number of $O(80)$ different fits. The first error is of statistical origin while the second, the asymmetric one, accounts for the systematic uncertainties. $\Sigma$ and $m_{u, d}$ are renormalised in the $\overline{\mathrm{MS}}$ scheme at the renormalisation scale $\mu=2 \mathrm{GeV}$, as the values of $Z_{\mathrm{P}}$ are in the $\overline{\mathrm{MS}}$ scheme at scale $2 \mathrm{GeV}$. The scale is set by $f_{\pi}=130.7 \mathrm{MeV}$

\begin{tabular}{l|c|c|c}
\hline \multicolumn{1}{c|}{ Quantity } & Median & Statistical & Systematic \\
\hline$m_{\text {up }, \text { down }}[\mathrm{MeV}]$ & 3.54 & $(19)$ & $(+16-17)$ \\
$\bar{l}_{3}$ & 3.50 & $(9)$ & $(+9-30)$ \\
$\bar{l}_{4}$ & 4.66 & $(4)$ & $(+4-33)$ \\
$f_{0}[\mathrm{MeV}]$ & 121.5 & $(0.1)$ & $(+1.1-0.1)$ \\
$r_{0}[\mathrm{fm}]$ & 0.420 & $(9)$ & $(+10-11)$ \\
$|\Sigma|^{1 / 3}[\mathrm{MeV}]$ & 270 & $(5)$ & $(+3-4)$ \\
$f_{\pi} / f_{0}$ & 1.0755 & $(6)$ & $(+8-94)$ \\
\hline
\end{tabular}



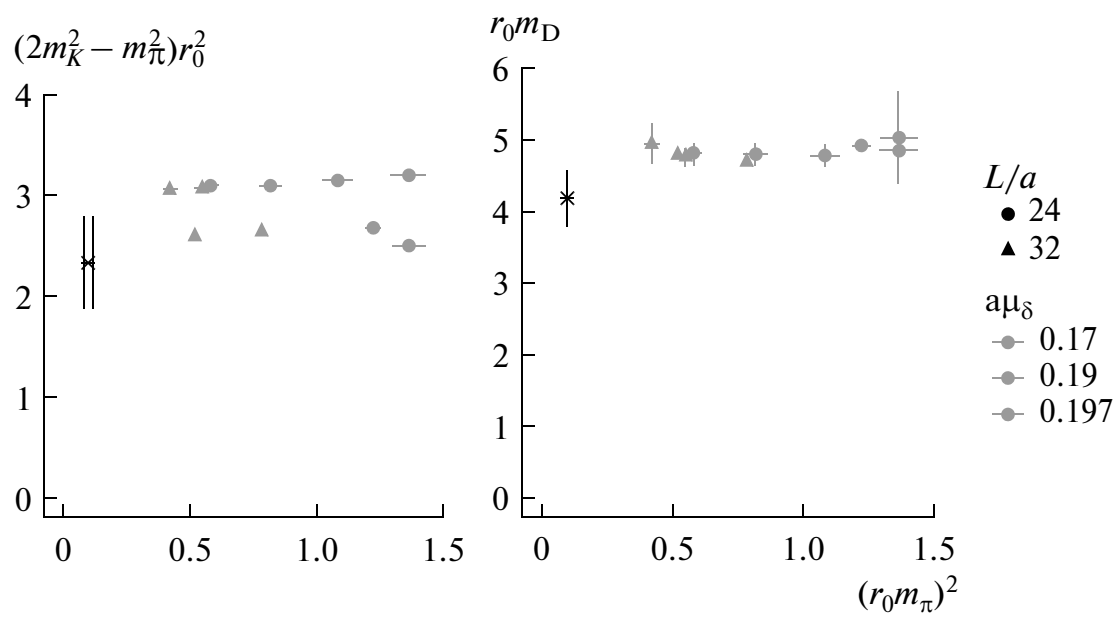

Fig. 3. $r_{0}^{2}\left(2 m_{K}^{2}-m_{\pi}^{2}\right)$ and $r_{0} m_{D}$ as functions of $\left(r_{0} m_{\pi}\right)^{2}$, showing the status of the tuning of the strange and charm quark mass respectively. The experimental value from PDG is added as the black cross $\left(r_{0}=0.44(4)\right.$ fm was used). Red points label the $\beta=$ 1.95 runs, green points label the $\beta=1.90$ runs, where the single blue point corresponds to $\beta=1.90$ with a different heavy sector splitting $a \mu_{\delta}$. Circles denote runs with $L / a=24$, triangles indicate a volume with $L / a=32$.

the low energy constants listed in Table 3 belong to the most precise determination of their kind world-wide.

\section{RESULTS ADDING DYNAMICAL STRANGE AND CHARM QUARKS}

The very nice results for mass degenerate quarks discussed in the last section motivates to go one step beyond this setup. The ETM collaboration has by now included the strange and the charm degrees of freedom in their simulations and they are the first collaboration to perform such studies.

The fermionic action for the light doublet is given by the same form as given in Eq. (1). In the heavy sector, the action becomes:

$$
\begin{gathered}
S_{h}=a^{4} \\
\times \sum_{x}\left\{\bar{\chi}_{h}(x)\left[D_{W}[U]+m_{0, h}+i \mu_{\sigma} \gamma_{5} \tau_{1}+\mu_{\delta} \tau_{3}\right] \chi_{h}(x)\right\}
\end{gathered}
$$

The heavy doublet mass parameters $\mu_{\sigma}$ and $\mu_{\delta}$ should be adjusted in order to reproduce the values of the renormalized strange $(s)$ and charm $(c)$ quark masses. The latter are related to $\mu_{\sigma}$ and $\mu_{\delta}$ via [18]:

$$
\left(m_{s, c}\right)_{\mathrm{R}}=\frac{1}{Z_{P}}\left(\mu_{\sigma} \mp \frac{Z_{P}}{Z_{S}} \mu_{\delta}\right)
$$

where the minus sign corresponds to the strange and the plus sign to the charm quark. In practice we fix the values $\mu_{\sigma}$ and $\mu_{\delta}$ by requiring the resulting $K$ - and $D$ meson masses to match experimental results.

Tuning to maximal twist requires to set $m_{0, l}$ and $m_{0, h}$ to a sufficiently accurate value of the critical mass $m_{\text {crit }}=m_{\text {crit }}(\beta)$ [18]. Here we set $m_{0, l}=m_{0, h} \equiv 1 /(2 \kappa)-4$. As has been shown in $[18,19]$, this is consistent with
$O(a)$ improvement defined by the maximal twist condition $a m_{\mathrm{PCAC}, l}=0$. We demand that the ratio $\left|Z_{\mathrm{A}} a m_{\mathrm{PCAC}} / a \mu_{q}\right| \lesssim$ 0.1 and also that its error $\Delta\left(Z_{\mathrm{A}} a m_{\mathrm{PCAC}} / a \mu_{q}\right) \lessgtr 0.1$. These criteria and their justification for tuning to maximal twist have been discussed in [7]. Note that we tune to $m_{\text {crit }}(\beta)$ at each value of $\mu_{q}$ separately.

A most interesting result in this $N_{f}=2+1+1$ setup is the calculation of the $K$ - and $D$-meson sector. Leaving out technical details of this complicated and demanding calculation, we show in the left panel of Fig. 3 the tuning of the strange quark mass by showing the difference, scaled with the chirally extrapolated value of $r_{0} / a$ between twice the $K$-meson mass squared and the pion mass squared. For our coarse value of the lattice spacing at $\beta=1.90$ (green points) the data appear to overshoot the physical point (the black cross on the left), while at our finer value of the lattice spacing at $\beta=1.95$ (red points) the $K$-meson mass extrapolates better. To improve the tuning of the strange quark mass, we are currently applying a reweighting procedure as described in [20] in the parameters $a \mu_{\delta}$ and $\kappa$.

In the right panel of Fig. 3 the mass of the $D$-meson as a function of the pion mass squared is shown for various simulation points as well as the experimental value from the Particle Data Group [16]. The plot demonstrates that we have tuned the charm (sea) quark mass in our simulations to a physically realistic value.

In the light meson sector we can perform a similar analysis as discussed above for the case of two massdegenerate flavours of quarks. Assuming that $Z_{P}$ is effectively a function of $\beta$ in the range of parameters we are considering, we can fit the ratio of those $Z_{P}$-values and lattice spacings and extract lattice spacings 
from the combined fit. In every fit we use as inputs the physical $f_{\pi}$ and $m_{\pi}$, and extract $f_{0}, \bar{l}_{3}$ and $\bar{l}_{4}$. As a result from a NLO $S U(2) \chi \mathrm{PT}$ fit, we find $f_{0}=121(4) \mathrm{MeV}$, $\bar{l}_{3}=3.5(2)$ and $\bar{l}_{4}=4.7(2)$. Comparing these results with those of Table 3 , we find a very good agreement between our $N_{f}=2$ and $N_{f}=2+1+1$ simulations. This fact and the comparison of $f_{\mathrm{PS}}$ and $m_{\mathrm{PS}}$ directly as done in [17] makes us confident that also for the situation of $N_{f}=2+1+1$ flavours of quarks the lattice spacing scaling violations will be small. We remark that by using $f_{\pi}=130.7 \mathrm{MeV}$ as our physical input parameter, we find from the fit a value of the lattice spacing of $a \approx 0.086$ at $\beta=1.9$ and $a \approx 0.078$ at $\beta=1.95$.

\section{CONCLUSIONS}

In this contribution we have discussed a particular formulation of lattice QCD, maximally twisted mass fermions. We have shown that with this $\widetilde{O}(a)$-improved action precise results in the light meson and the strange baryon sector can be obtained. In the course of this investigation, it has been demonstrated that twisted mass fermions at maximal twisted indeed scale with $a^{2}$ towards the continuum limit and that even these remaining $a^{2}$ corrections are very small giving rise to a well controlled continuum limit extrapolation of lattice results. Fitting the data for $f_{\pi}$ and $m_{\pi}$ it became possible to extract a number of physical quantities and low energy constants of chiral perturbation theory very precisely, see Table 3.

Encouraged by these results, we have described first simulations for the situation when a dynamical strange and charm quark are included. We have demonstrated that it is possible to tune to physical $K$ - and $D$-meson masses. In addition the good agreement of the $N_{f}=2$ and $N_{f}=2+1+1$ simulations, visible when comparing the values for the low energy constants $f_{0}, \bar{l}_{3}$ and $\bar{l}_{4}$ with those of Table 3 indicates that also for the latter case lattice artefacts, at least in the light quark sector, are small. This offers the promising possibility to obtain precise physical results also with dynamical up, down, strange and charm simulations for the first time.

\section{ACKNOWLEDGMENTS}

We thank all other members of the ETM Collaboration for a very fruitful collaboration and many valu- able discussions. The HPC resources for this project have been made available by the computer centres of Barcelona, Groningen, Jülich, Lyon, Munich, Paris and Rome (apeNEXT), which we thank for enabling us to perform this work. This work has also been supported in part by the DFG Sonderforschungsbereich/Transregio SFB/TR9-03, and by GENCI (IDRIS CINE S), Grant 2009-052271.

\section{REFERENCES}

1. C. Alexandrou et al., arXiv:0910.2419 [hep-lat].

2. K. G. Wilson, Phys. Rev. D 10, 2445 (1974).

3. R. Frezzotti, P. A. Grassi, S. Sint, and P. Weisz (ALPHA Collab.), J. High Energy Phys. 08, 058 (2001), hep-lat/0101001.

4. R. Frezzotti and G. C. Rossi, J. High Energy Phys. 10, 070 (2004), hep-lat/0407002.

5. P. Boucaud et al. (ETM Collab.), Phys. Lett. B 650, 304 (2007), arXiv:hep-lat/0701012.

6. B. Blossier et al. (ETM Collab.), J. High Energy Phys. 04, 020 (2008), arXiv:0709.4574 [hep-lat].

7. P. Boucaud et al. (ETM Collab.), Comput. Phys. Commun. 179, 695 (2008), arXiv:0803.0224 [hep-lat].

8. R. Baron et al. (ETM Collab.), arXiv:0911.5061 [hep-lat].

9. R. Frezzotti and G. C. Rossi, J. High Energy Phys. 08, 007 (2004), hep-lat/0306014.

10. P. Dimopoulos et al. (ETM Collab.), arXiv:0908.0451 [hep-lat].

11. R. Sommer, Nucl. Phys. B 411, 839 (1994), heplat/9310022.

12. S. Weinberg, Physica A 96, 327 (1979).

13. J. Gasser and H. Leutwyler, Ann. Phys. 158, 142 (1984)

14. J. Gasser and H. Leutwyler, Nucl. Phys. B 250, 465 (1985).

15. G. Colangelo, S. Dürr and C. Haefeli, Nucl. Phys. B 721, 136 (2005), hep-lat/0503014.

16. C. Amsler et al. (Particle Data Group), Phys. Lett. B 667, 1 (2008).

17. R. Baron et al. (ETM Collab.), arXiv:0911.5244 [heplat].

18. R. Frezzotti and G. C. Rossi, Nucl. Phys. Proc. Suppl.128, 193 (2004).

19. T. Chiarappa et al., Eur. Phys. J. C 50, 373 (2007).

20. R. Baron et al. (ETM Collab.), PoS LAT2008, 094 (2008). 